above. Colonel $\mathrm{Y}$ - recovered with a useful arm, but the prominence remained to a certain degree.

3. Mr. P-, jun., aged eighteen, fell on the point of his shoulder (side not mentioned), and was supposed to have dislocated the bead of his humerus. The supposed dislocation was twice reduced, but failed to remain in position. Three weeks after the injury he was seen by Mr. Guthrie. The elbow was carried very far back wards and the head of the humerus forwards; the prominent head did not feel like the normal head, but seemed as if it had been split. After bleeding and tartar emetic, reduction by pulleys twice failed, and after this the position was somewhat improved. The outer part of the head seemed in the glenoid cavity, but the inner and greater part was internal to it. The elbow was in proper position, and the arm could be pressed against the ribs. He recovtred with a useful arm.

Fourteen years later Dr. R. W. Smith, of Dublin, in his work "Fractures in the vicinity of Joints," (1847, p. 176) directed attention to simi'ar injuries, but differed from Guthrie as to their explanation. He called them fractures of the greater tuberosity, and stated the following appearances as being characteristic of them. They resemble dislocations in the prominence of the acromion, in the altered contour of the shoulder, impaired motion, separation of the arm from the side, and ociasional absence of crepitus; but differ from them in the increased breadth of the articulation, which is sometimes twice the natural breadtb, and in the projection of the acromion being less than in luxation. The rounded form of the shoulder is altered and the deltoid somewhat flattened, but the finger cannot be sunk into the glenoid cavity; the arm can be without difficulty approximated to the side; the "under" motions of the limb can be per. formed, but the patient cannot raise it beyond the horizontal line, and seldom even so far; and he supports the injured arm with the opposite hand. The articulation presents two osseous tumours, the one externally beneath the acromion, and the other, the larger, beneath and a little internal to the coracoid process. The latter feels like the rounded head of the bone, moves with the arm, and exhibits a sulcus of varying depth and distinctness between it and the other fragment in the situation of the bicipital groove. Dr. Smith brings forward three cases, one of them, quoted as having been communicated to the editor of Sir Astley Cooper's work on Dislocations by Mr. Herbert Mayo, having no resemblance to Gutbrie's cases, and, as it was in all probability (the details are most meagre) a fracture of the greater tuberosity alone, it does not concern the injury in hand. The other two cases are original observations.

The first, (4) was discovered at the post-mortem of Julia D-, aged eigbty. The left shoulder had, to a certain extent, lost its rounded form. The acromion was prominent, but less so than in dislocation. The breadth of the articulation was greatly increased; and, on pressure beneath the acromion, an osseous tumour, not like the head of the humerus, but moving with the arm (the injury was ancient), could be felt in the glenoid cavity through the soft parts. $\Lambda$ second and larger tumour, presenting the rounded form of the head of the humerus, lay beneath the level of, and internal to, the coracoid process; and between the two the finger could be sunk into a deep sulcus, placed immediately below the coracoid process. The elbow could be brought into contact with the side, and there was no appreciable alteration in the length of the arm. On dissection, the head of the humerus lay beneath and partly internal to the coracoid process. The greater tuberosity, with a small portion of the outer part of the head, had been completely separated from the shaft. This portion occupied the glenoid cavity, and was contained within the capsular ligament. The fracture traversed the upper part of the bicipital groove. A new and shallow socket had been formed on the costal surface of the neck of the scapula below the root of the coracoid process, and the inner edge of the glenoid cavity corresponded with the posterior part of the sulcus which separated the head of the bone from the detached tuberosity. The latter was united to the shaft only by ligament. The articular capsule had not been injured, but was enlarged, thickened, and partly ossified. No history could be obtained. In the woodcut illustrating this case Dr. Smith has depicted the biceps tendon as lying in the sulcus formed by the fracture.

The other case (5) was that of a man, aged thirty, who fell from a house three storegs high, and received a severe injury of the left shoulder-joint. When taken to Richmond Huspital, the head of the humerus was placed more forwards than natural, the acromion more prnminent, the deltoid somewhat flattened, and the elbow separated from the side. Reduction, by the knee in the arilla, of the supposed dislocation was had recourse to, the deformity apparently removed, and the arm bandaged to the side. In a short time the bandages were removed for severe pain, and the deformity was seen to have returned. On examination crepitus was detected. Next day he was seen by Dr. Smith, in consultation with Mr. Adams. The limb presented appearances similar to those in the preceding case. The acromion was prominent, but the finger could not be sunk into the glenoid cavity. There was no diffeulty in approximating the arm to the side; the breadth of the joint was nearly double that of the opposite one; and two tumours were distinctly noticed, the inner and larger of which, placed under the coracoid process, was evidently constituted by the head of the humerus, while the external and smaller apparently formed by the greater tuberosity, corresponded in situation to the glenoid cavity; they were separated by a deep and well-marked sulcus, which followed the direction of the bicipital groove. The deformity could not be completely removed by any means whatever.

These two cases of Dr. Smith's are evidently what he claimed them to be-fracture of the greater tuberosity with more or less of the adjoining portion of the head of the bone; in other words, obliquely longitudinal fracture of the head of the bumerus, the fracture extending in the direction of the bicipital groove and the greater part of the head renaining attached to the lesser tuberosity. M (Holmes"s " System of Surgery," 1870, vol. ii., p. 782) refer Dr. Smith's cases to the category of dislocations complicated with fracture of the great tuberosity; but there seems every reason to regard this opinion as erroneous, and to agree in the view taken of them by Dr. Smith himself. They plainly have not the least analogy with the tealing off of the bone at the points of muscular insertion into the great tuberosity -so common a complication of dislocations of the shoulderjoint. Dr. Smith claims Guthrie's cases as being of the same nature as bis own, and certainly one of them (No. 3 of the present paper) seems exactly similar, the greater part of the head of the humerus having heen felt in connexion with the lesser tuberosity. But Gntbrie's other two cases (Nos. 1 and 2 of the present paper) deserve to be looked upon in the ligbt in which he himself regarded them-as oblique fractures where the whole or the greater part of the head remained attached to the great tuberosity; for it is expressly mentioned that the projection in the situation of the lesser tuberosity felt rough, irregular, and unlike the head, and that the greater part of the head could be felt in the glenoid cavity. (To be concluded.)

\section{THE FORMATION OF LAMINATED CLOT.}

\section{BX RALPH W. LEFTWICH, M.D., C.M., M.R.C.S.}

The views which have hitherto been advanced respecting the formation of this variety of clot are, briefly, that it is(1) a malignant growth; (2) the result of an inflammation of the sac with successive effusions of $l y \mathrm{mph}$; (3) a subsequent stratification of passive clot; (4) due to alternate agitation and comparative stagnation of the blood in the sac (Broca). Of these, the first is, of course, quite obsolete; the second and third are incompatible with the fact that the outer laminæ are older than the inner; and the fourth fails to explain its occurrence in situations where the blood is always still. The view now brought forward is at least open to none of these objections.

The deposition of the first layer in an aneurism presents no diffculties; since it is admitted that the expanded, thickened, and perhaps detached wall of the artery sufficiently resembles a foreign body to account for this. The deposit, however, must be of limited thickness, for the soft coat of fresh clot will soon hide the roughness and present to the blood a surface as smooth as that of the lining mem. 
brane of a healthy artery. Thus, as soon as this point is reached, the process is interrupted. What happens now is not very mysterious : it is simply that this thin layer of clot follows its natural tendency and contracts; and that, in the contracted state, it once more presents a rough surface ready for the deposition of a freeh layer.

It is necessary here to examine more carefully the way in which it contracts. Contraction of the lamina may take place in two principal directions-that of its long, and that of its short axis; by "long axis" must be understood the direction of its length or breadth; by "short axis," the direction of its thickness. The former alone can reduce the size of the cavity, the latter will slightly enlarge it. Now contraction in the long axis is prevented from occurring at present by the pressure of the blood; the roughened surface is due, therefore, to contraction in the direction of its thickness alone.

The otber layers are deposited in the same way, each in its turn contracting so as to form the roughened surface ready for the reception of the next. Thus a definite interval of time occurs after each deposit, and the record of this interval is found in the stratum of leucocytes which occupies the inner aspect of each lamina; for during the process of contraction, these corpuscles, in hugging the sides, have either become entangled in the clot or have partially migrated into it.

It may be questioned whether the contraction of a layer can make its surface sufficiently uneven for fibrin to be deposited upon it. Indeed, it is evident that in some cases it is inadequate, otherwise all aneurisms wonld be cured. One other condition, therefore, is necessary-viz,, a moderate degree of rest. That, when conjoined with rest, it suffices, has been proved by Durante (quoted in Brücke's Vorl. ü. Physiologie), who showed that even microscopic degrees of roughness were sufficient to produce deposition of bloodclot.

Iaminated fibrin has been found in many other situations, but the explanation given of its production in aneurisms may readily be applied to it elsewhere. It will be well, however, to consider some of these in detail.

The conical clot in a ligatured artery is composed of this substance. When an artery is deligated, the blood first clots upon the shrivelled retracted end, and forms a layer of limited thickness. This then contracts, and admits of the reception of a second. Others follow, until the first branch is reached, when, the important auxiliary condition, rest, being no longer present, further increase is prevented. The conical shape is thus explained. There is here no obstacle to contraction in the long axis. The first layer, therefore, contracts in both directions; then the second layer is deposited; but this occupies, when fresh, the same extent of surface as its predecessor when contracted; so that, after its own contraction, its superficies is smaller. Each layer, therefore, being smaller than the one before, the shape of the whole mass is necessarily conical. It is not easy to account for the presence of the canal which is sometimes found in the centre of the internal clot, unless it be assumed that, in these cases, the artery was diseased, and that the rough surface of the tunica intima was the seat of the first lamina: the process being continued in the way described below as true of certain blood-cysts. The increase in the size of an embolus after its impaction occurs in precisely the same manner as that described for conical clots, except that if the blood have access to it on both sides it will grow in both directions.

This form of fibrin is frequently found upon the valves of the diseased heart, and is here a fruitful source of syst emic emboli. The size it attains is necessarily limited, on account of the motion of the blood (there being no real analngy between this and "whipping"); but while in a healthy heart rest would probably be entirely absent, yet in a diseased condition, and especially in stenosis, it would be doubtless present in some degree.

The occurrence in certain blood-cysts of sernm in the interior of the clot can be readily explained without having recourse to such a chemically improbable idea as the breaking down of the centre of the clot-i.e., the conversion of fibrin into serum. It is well-known that blood effused will remain flind for a long time. When therefore the blood-cyst is first formed a thin layer is deposited upon the walls of the carity. This then contracts, chiefly in its short axis, and other layers are superposed in turn. But, since the first lamina effectually prevent the further effusion of blood, there comes a time when all the fibrin contained in the cyst is exhausted, and the broken-down corpuscles being in great measure included in the clots, nothing remains but some blood-stained serum. Laminated fibrin has been found in bæmatoceles. In such cases, the formation of the first layer may have been due to the fibrino-genetic action of the fluid secreted by the tunica vaginalis.

In the beginuing of this paper it was shown that the obstacle to contraction in the long axis of the lamina is the pressure of the blood. Now, when an aneurism is treated surgically, this is almost completely abolished. The thickened sac of the aneurism, formed as it is of new fibrous tissue, is in a condition precisely analogous to that of the laminæ. The whole contracts therefore as one mass, and the cavity is lessened or obliterated. The important part played in the cure of aneurism by the contraction of the sae has long been recoguised, but, if this view be correct, it is even more important than has been supposed. That very little clot is necessary for the cure of an aneurism is shown by a case of Mr. Maunder's, where, five months after ligature, the diseased artery was found of the same calibre tbroughout, and the seat of aneurism could not be detected. ("Surgery of the Arteries," p. 66.)

Mr. Holmes argues, from the occasional rapid cure of aneurism (e.g., by digital pressure), that there is not time for the formation of laminated clot, and that consequently in some cases passive clot must subsequently become stratified. With great respect for the opinion of one who is so great an autbority upon aneurism, it is submitted that the large amount of contractile force thus suddenly brought into use may suffice to explain it.

It is interesting to observe in some specimens bow this contractile force of the clot, increasing with each additional layer, becomes at length sufficient to counterbalance the blood pressure, and how this pressure is then exerted suecessfully upon the unprotected surface of the distal side of the sac. Many cases of aortic aneurism involve the innominate artery in this way. In the distal operation for aneurism the occasionally successful result is due probably to the fact that the blood, following the course of least resistance, exerts less pressure than formerly upon the dilatation. This would be especially true in those cases where, owing to the position of the vessel, the sac remains full during the diastole. It is suggested that, in certain cases of internal aneurism, all the benefit of the distal operation in its most favourable conditions may be obtained by placing the patient in such a position that the orifice of the sac be uppermost. The blood would thus be at rest, and its active pressure would not be directed against the walls of the cavity. The situation of the orifice may generally be easily determined by auscultation; for if the sac remain full during the diastole, the bruit will either have disappeared or have been considerably diminished.

The principle of all treatment therefore must be to diminish blood-pressure. This may be accomplished by rest in the horizontal position, low diet, and removal from all sources of excitement, and by venesection. Local means are ligature or compression of arteries, special decubitus, and even galvano-puncture, or other methods for the produetion of passive clot in the sac. Of drugs, those which lower blood-pressure would be indicated; but since the action of these is rarely uncomplicated, care should be used in their administration.

Hyoscyamin in Chorea-Dr. Oulmont has been very successful in five cases of chorea, treated by him with hyoscyamin in his wards of the Hôtel Dieu. In one case the patient was two months pregnant. Various drugs had failed, and notably bromide of potassinm in doses of three drachms daily. Hyoscyamin was given in gradually increasing doses of from two to eight milligrammes daily. Amelioration was soon evident, and the case speedily got well.

Eartswood Idiot Asylum.-The twenty-ninth anniversary festival of this excellent institation was held on Weduesday, at the $L$ indon Tavern. The chair was occupied by the Earl of Shaftesbury, and a numerons and distinguished company were drawn together on the occasion. During the evening the secretary announced the receipt of subscriptions amounting to upwards of $\$ 2400$. 